\title{
ROBUST ADAPTIVE ARRAY BEAMFORMING IN TWO DIMENSIONS
}

\author{
Ju-Hong Lee and Jeng-Hong Chen \\ Department of Electrical Engineering \\ National Taiwan University \\ Taipei, Taiwan, R.O.C.
}

\section{ABS'IRACT}

An effective technique for robust adaptive beamforming using one-dimensional (1-D) linear array has been proposed by one of the authors in [1]. It was shown that the resulting hountomer can cure the difficulties of using conventional robust techuiques. In this paper, we investigate the extension of the above technique to two dimensions (2-D). We present two algerithms for the implementation of the 2-D robust technique. The comparison based on computational complexity is made. Computer simulations are given for illustration.

\section{INTRODUCTION}

Adaptive array beamformer designed for receiving the desired signal in specified beam direction while rejecting noise and other directional jammers is widely used in radar, sonar, and many communication systems. However, its performance is very sensitive to the accuracies of signal and array situations because an optimum adaptive beamformer is designed under the assumptions of ideal signal characteristics and exact array configurations. It has been shown that the effectiveness of an alaptive beamformer can be destroyed even if a small. mismatch arises in practical circumstances.

Many techniques have been presented to tackle the degradation effect due to steering errors [2-9]. These conventional methods are based on either imposing directional derivative constraints to allow direct control of array scusitivity or reducing the sensitivity of array performance to the steering errors. However, the drawback is that the abilities of eliminating noise and interference are inevitably deteriorated. Recently, one of the authors has proposed an alfective approach for robust adaptive beamforming in [1] to deal with the case of using 1-D linear array. In practice, it is possible l, hat there exists more than one signal coming from different directions but having the same angle with respect to the broadside of a 1-D linear array. In this circumstances, the
1-D array cannot distinguish these different signals. Hence, $2-\mathrm{D}$ arrays are required for the purpose of beamforming. In this paper, We extend the technique of [1] to two dimensions. It is shown that the resulting 2-D adaptive array beamformer is robust in the presence of steering errors.

\section{THE 2-D ROBUST ADAPTIVE BEAMFORMING}

The 2-D array is assumed to be rectangular with size $\mathrm{M} \mathrm{x} \mathrm{N}$ and the array sensors are equally spaced with interelement spacings along the $X$ and $Y$ axes are $d_{x}$ and $d_{y}$, respectively. Its configuration is shown in Figure 1. The directional angles of the $\mathrm{j}$-th jammer and the signal are denoted by $\left(\theta_{\mathbf{k}}, \phi_{\mathrm{k}}\right), \mathrm{k}=1,2, \ldots, \mathrm{K}$ and $\left(\theta_{\mathrm{s}}, \phi_{\mathrm{s}}\right)$, respectively. The input of the mn-th array sensor, which is located at the intersection of the $\mathrm{m}$-th row and the $\mathrm{n}$-th column, is given as

$$
\begin{aligned}
\mathrm{u}_{\mathrm{mm}}(\mathrm{t}) & =\alpha_{\mathrm{s}}(\mathrm{t}) \exp \left[j \mathrm{k}_{\mathrm{c}}\left(\mathrm{md}_{\mathrm{x}} \mathrm{u}_{\mathrm{s}}+\mathrm{nd}_{\mathrm{y}} \mathrm{v}_{\mathrm{s}}\right)\right] \\
& +\sum_{\mathrm{j}=1}^{\mathrm{K}} \alpha_{\mathrm{j}}(\mathrm{t}) \exp \left[j \mathrm{k}_{\mathrm{c}}\left(\mathrm{md}_{\mathrm{x}} \mathrm{u}_{\mathrm{j}}+\mathrm{nd}_{\mathrm{y}} \mathrm{v}_{\mathrm{j}}\right)\right]+\mathrm{n}_{\mathrm{mn}}(\mathrm{t})
\end{aligned}
$$

where $\alpha_{s}(t)$ and $\alpha_{j}(t)$ denotes the complex envelopes of the desired signal and the $j$-th jammer, respectively, $k_{c}$ the wavenumber, and $u_{s}=\sin \theta_{s} \cos \phi_{s}, v_{s}=\sin \theta_{s} \sin \phi_{s}, u_{j}=\sin \theta_{j} \cos \phi_{j}$, $v_{j}=\sin \theta_{j} \sin \phi_{j}$, and $n_{m n}(t)$ is the complex noise of $m n-t h$ sensor. Using (1), the input signal vector of the m-th row of the 2-D array, which is of size $\mathrm{N} \times 1$, is given as

$$
\begin{aligned}
\underline{U}_{m n}(t) & =\left[u_{m 1}(t), \ldots, u_{m_{N}}(t)\right]^{T} \\
& =\alpha_{s}(t) \underline{s}_{s m}+\sum_{j=1}^{K} \alpha_{j}(t) \underline{s}_{j m}+\underline{n}_{m}(t)
\end{aligned}
$$

where $\underline{s}_{s m}$ is the signal phase vector at the m-th row, 


$$
\begin{aligned}
\underline{s}_{s m}= & {\left[s_{s m 1}, \ldots, s_{s m N}\right]^{T}=\left[\exp \left[j k_{c}\left(m d_{x} u_{s}+d_{y} v_{s}\right)\right] \ldots\right.} \\
& \left., \exp \left[j k_{c}\left(m_{x} u_{s}+N d_{y} v_{s}\right)\right]\right]^{T}
\end{aligned}
$$

$\underline{s}_{j m}$ is the phase vector of the $j-$ th jammer, and $n_{m}(t)$ is the noise vector. The input vector of the $2-D$ rectangular array can be defined as the $\mathrm{MN} \times 1$ vector given by

$$
\begin{aligned}
\underline{\mathrm{U}}(\mathrm{t}) & =\left[\underline{\mathrm{U}}_{1}(\mathrm{t})^{\mathrm{T}}, \ldots, \underline{\mathrm{U}}_{\mathbf{M}}(\mathrm{t})^{\mathrm{T}}\right]^{\mathrm{T}} \\
& =\alpha_{\mathrm{S}}(\mathrm{t}) \underline{\underline{S}}_{\mathrm{S}}+\sum_{\mathrm{j}=1}^{K} \alpha_{\mathrm{j}}(\mathrm{t})_{\underline{\mathrm{S}}_{\mathrm{j}}}+\underline{\mathrm{n}}(\mathrm{t})
\end{aligned}
$$

where $\underline{s}_{s}$ is the phase vector of the desired signal

$$
\underline{s}_{s}=\left[\underline{s}_{s}{ }_{s}^{T}, \ldots, \underline{s}_{s_{M}}^{T}\right]^{T}
$$

and $n(t)$ is the noise vector. Then the covariance matrix is given as

$$
\mathrm{R}=\mathrm{E}\left[\underline{\mathrm{U}}(\mathrm{t}) \underline{\mathrm{U}}^{\dagger}(\mathrm{t})\right]
$$

where $\dagger$ denotes the conjugate transposition and $E$ the expectation.

Consider the $\mathrm{m}-$ th row of the $2-\mathrm{D} \mathrm{M} \times \mathrm{N}$ array. Assume that the tolerance error of the steering error, which is given as $s_{d m}$ with steering angle $(\theta, \phi)$, due to the mismatch is smaller than the distance between steering and jammer phase vectors. Based on the fact that the output signal-to-noise ratio (SNR) of an optimized adaptive beamformer becomes maximum when the steering vector concides with the signal phase vector, we first find the optimum weight vector $\underline{W}_{\text {on }}^{\prime}$ for a given steering vector by minimizing the array output power with main beam constraint. After obtaining the $\underline{W}_{o m}^{\prime}$, we adjust the steering vector in a prescribed class $S_{c}$ of possible arrival phase vectors of the signal. This procedure is repeated until the output SNR of the optimized adaptive beamformer reaches a maximum. Therefore, the resulting optimization problem can be written as [1]

$$
\begin{array}{cl}
\operatorname{Maximize} & \text { Mnimize } \left.E\left(\left|\underline{W}_{m}^{\dagger} \underline{U}_{m}(t)\right|^{2}\right)\right\} \\
\underline{s}_{d m} \in S_{c} & \underline{W}_{m}^{\dagger} \underline{d}_{\mathrm{dm}}=1
\end{array}
$$

Subject to $\left|\mathrm{s}_{\mathrm{dmi}}\right|=1$, for $\mathrm{i}=1,2, \ldots, \mathrm{N}$

where $S_{c} \equiv\left\{\underline{s}_{d m} \mid\left\|\underline{s}_{d m}-\underline{s}_{s m}\right\|^{2} \leq \delta\right\}, \delta$ is the tolerance error for robustness. Therefore, we correct the steering vector error by iteratively adjusting the steering vector during the optimization process. The adaptive algorithm presented in [1] for 1-D robust adaptive beamforming can be employed to search the correct steering vector $\underline{S}_{s m}$. Then the corresponding optimum weight vector is given as $[10]$

$$
\underline{W}_{\mathrm{om}}=R_{\mathrm{um}}^{-1} \underline{s}_{\mathrm{sm}} / \underline{s}_{\mathrm{sm}}^{\dagger} R_{\mathrm{um}}^{-1} \underline{s}_{\mathrm{sm}}
$$

for $\mathrm{m}=1,2, \quad, \mathrm{M}$, where $\mathrm{R}_{\mathrm{um}}=\mathrm{E}\left[\mathrm{U}_{\mathrm{m}}(\mathrm{t}) \underline{\mathrm{U}}_{\mathrm{m}}^{\dagger}(\mathrm{t})\right]$. Finally, all of the M resulting 1-D optimum weight vectors are used to form an $\mathrm{MN} \times \mathrm{l}$ array weight vector $\underline{\mathrm{W}}_{0}$ which is given as

$$
\underline{W}_{0}=\left[\underline{w}_{01}^{T}, \ldots, \underline{w}_{0 M}^{T}\right]^{\mathrm{T}}
$$

and then obtain the associated 2-D array beam pattern.

An alternative approach is to only save the corrected steering vector $\underline{s}_{s m}$ after the $1-D$ robust processing. Then all of the $\mathrm{M}$ corrected steering vectors are used to form an $\mathrm{MN} \times 1$ steering vector as in (5). Then we compute the corresponding $\mathrm{MN} \times 1$ array weight vector $\underline{W}_{0}$ which is given as

$$
\underline{W}_{0}=R^{-1} \underline{s}_{s} / \underline{s}_{s}^{\dagger} R^{-1} \underline{s}_{s}
$$

Comparing the computational complexity, we note from (8) and (10) that the second algorithm faces the problem of computing the inverse of $\mathrm{R}$ with size $\mathrm{MN} \times \mathrm{MN}$ in (10). This leads to heavy computational burden. By contrast, using the first algorithm requires the computation of inverse of $\mathrm{R}_{u m}$ with size $\mathrm{N} \times \mathrm{N}$ only in (8). In the next section, we thus only present the simulation examples based on the first algorithm.

\section{III.SIMULATION EXAMPLES}

In this section, several computer simulations are presented for illustration. We consider a $7 \times 72-D$ rectangular array. Both the interelement spacings $d_{x}$ and $d_{y}$ are set to be half-wavelength $(\lambda / 2)$. The desired signal is at $\left(\theta_{s}, \phi_{S}\right)=\left(0^{0}, 0^{0}\right)$ while two incoherent jammers are located at $\left(\theta_{1}, \phi_{1}\right)=\left(65^{0}, 20^{\circ}\right)$ and $\left(\theta_{2}, \phi_{2}\right)=\left(45^{0}, 40^{\circ}\right)$, respectively. The noise received at the array sensors is assumed to be spatially white with $\mathrm{SNR}=20$ $\mathrm{dB}$ and $\mathrm{JNR}$ (jammer-to-noise ratio) $=10 \mathrm{~dB}$.

\section{A. Example of Steering Angle Mismatch.}

In this case, the steering angle error is $(\Delta \theta, \Delta \phi)=\left(0.5^{0}, 0.5^{0}\right)$. Figure 2 shows the $2-\mathrm{D}$ array beam patterns under the steering angle error and using the proposed robust technique after 500 iterations, respectively. The ideal result for this case is shown in Figure 3. Table 1 lists the array gains for this case. Comparing these results, we note that the proposed robust technique can effectively cure the 2-D array performance degradation due to steering angle error. Moreover, the beam pattern using the robust technique is as good as the ideal beam pattern. 
B. Example of Sensor Position Error

In this case, we assume that the steering vector error is only due to 2-1D random perturbation in sensor positions. The random position errors are Gaussian with mean $=0$ and variance $=0.15$ half-wavelength in each dimension. Figure 4 shows the resulting 2-D array beam pattern when the 2-D array sulfers the $2-D$ random perturbation and the resulting 2-D array beam pattern using the proposed robust approach after 500 iterations, respectively. Table 2 lists the array gains for this case. Again, we note from these results that the proposed robust technique demonstrates its effectiveness in dealing with the degradation effect due to sensor position mismatch and provides a $2-D$ beam pattern as good as that of the ideal one shown in Figure 3.

\section{REFERENCES}

[1] Ju-Hong Lee and Jeng-Fu Wu, " A Novel Approach for Robust Adaptive Beamforming ", in Proc. of IEEE Int. Conf. on ASSP, Glasgow, UK, May 1989, pp. 2740-2743.

[2] F. Habor, Y. Bar-Ness, and C.C. Yeh, " An Adaptive Interference Canceling Array Utilizing Hybrid Techniques " IEEE Trans. AES, Vol AES-19, No.5, pp. 795-803, Sept. 1983.

[3] R.T. Compton, Jr. " The Effect of Random Steering Vector Errors in the Applebaum Adaptive Array ", IEEE Trans. AES, Vol AES-18, No.5, pp.392-400, Sept. 1982.

[4] R.T. Compton, Jr. " Pointing Accuracy and Dynamic Range in a Steered Beam Adaptive Array ", IEEE Trans. Vol. AES--16, No.3,pp. 280-287, May 1980.

[5] L.C. Codara, " Error Analysis of the Optimal Antenna Array Processors ", IEEE Tran. AES, Vol. AES-22, No.4, pp. 395-409, July 1986.

[6] N.K. Jablon, "Adaptive Beamforming with the Generalized Sidelobe Canceller in the Presence of Array Imperfections ", IEEE Trans. AP,Vol. AP-34, pp. 996-1012, Aug. 1986.

[7] M.H. Er and A. Cantoni, "Derivative Constraints for Broadband Element Space Antenna Array Processors ", IEEE Trans. ASSP, Vol. ASSP-31, No.6, pp. 1378-1393, Dec. 1983.

[8] K.M. Buckley and L.J. Griffths, " An Adaptive Generalized Sidelobe Canceller with Derivative Constraints ", IEEE Trans. AP, Vol. AP-34, No.3, pp. 311-319, Mar. 1986.

[9] K. Takao and N. Kikuma, "Tamed Adaptive Antenna Array ", IEEE Trans. AES, Vol. AES-34, No.2, pp.388-394, Mar. 1986.
[10] R.A. Monzingo and T.W. Miller, INTRODUCTION TO ADAPTIVE ARRAYS, New York: Wiley, 1980.

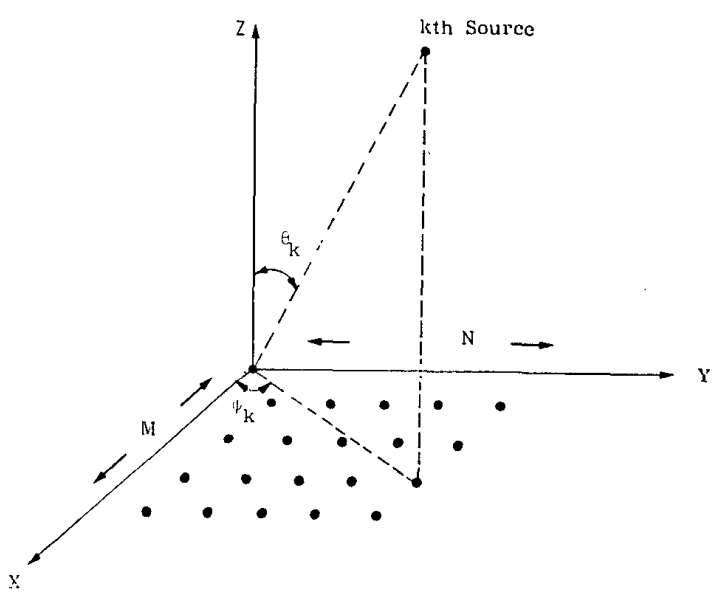

Figure 1 The 2-D MxN Rectangular Array

Table 1 Array Gains ( $d B$ ) for the Case of Steering Angle Error

\begin{tabular}{|l|l|l|l|}
\hline $\begin{array}{l}\text { signal } \\
\text { position } \\
\text { array } \\
\text { situation }\end{array}$ & $\left(0^{0}, 0^{0}\right)$ & $\left(65^{0}, 20^{\circ}\right)$ & $\left(45^{0}, 40^{\circ}\right)$ \\
\hline $\begin{array}{l}\text { Ideal } \\
\text { Array }\end{array}$ & 0.0 & -86.7 & -90.8 \\
\hline $\begin{array}{l}\text { Array with } \\
\text { Angle Error }\end{array}$ & -23.9 & -71.2 & -53.2 \\
\hline $\begin{array}{l}\text { Array after } \\
\text { Robust Method }\end{array}$ & 0.0 & -84.1 & -74.0 \\
\hline
\end{tabular}

Table 2 Array Gains (dB) for the Case of Position Error

\begin{tabular}{|l|l|l|l|}
\hline $\begin{array}{l}\text { Signal } \\
\text { position } \\
\text { array } \\
\text { situation }\end{array}$ & $\left(0^{\circ}, 0^{\circ}\right)$ & $\left(65^{\circ}, 20^{\circ}\right)$ & $\left(45^{\circ}, 40^{\circ}\right)$ \\
\hline $\begin{array}{l}\text { Ideal } \\
\text { Array }\end{array}$ & 0.0 & -64.0 & -74.5 \\
\hline $\begin{array}{l}\text { Array with } \\
\text { Position } \\
\text { Error }\end{array}$ & 0.0 & -21.2 & -22.6 \\
\hline $\begin{array}{l}\text { Array after } \\
\text { Robust Method }\end{array}$ & 0.0 & -63.0 & -73.5 \\
\hline
\end{tabular}




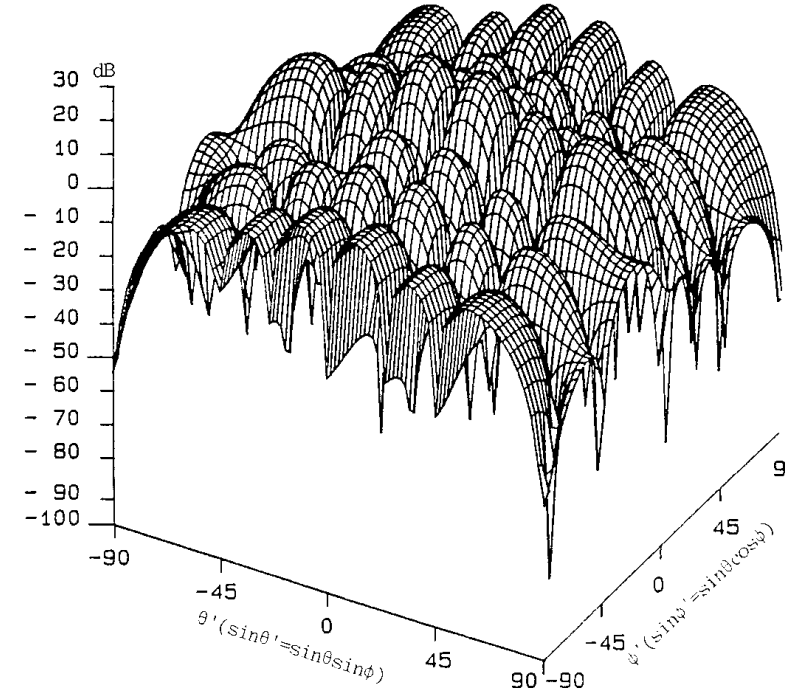

Figure 2 (a) The Array Beam Pattern in The Case of Steering Angle Error

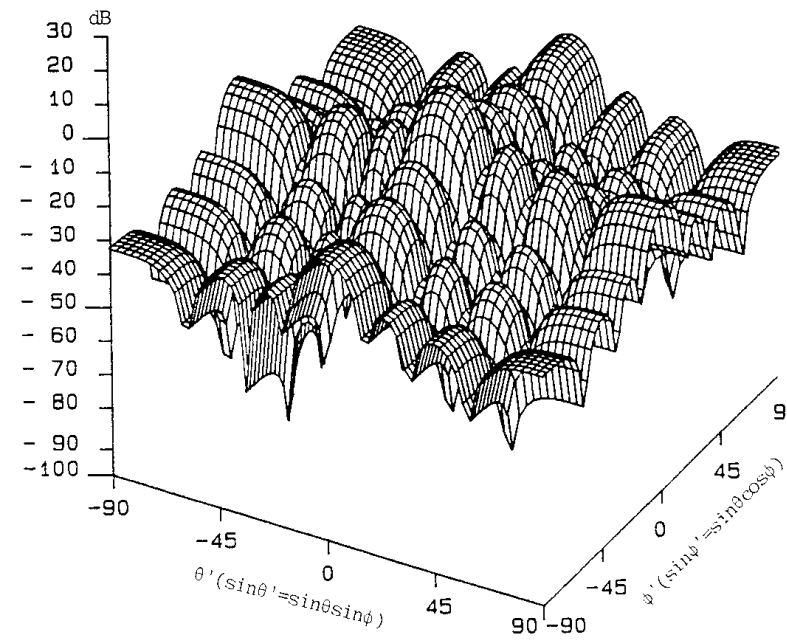

Figure 2 (b) The Array Beam Pattern After Robust Processing

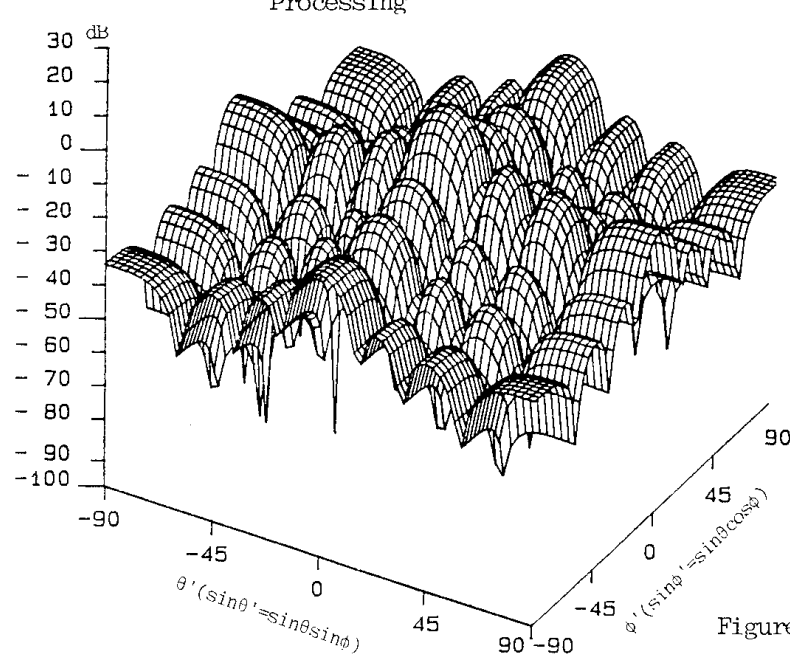

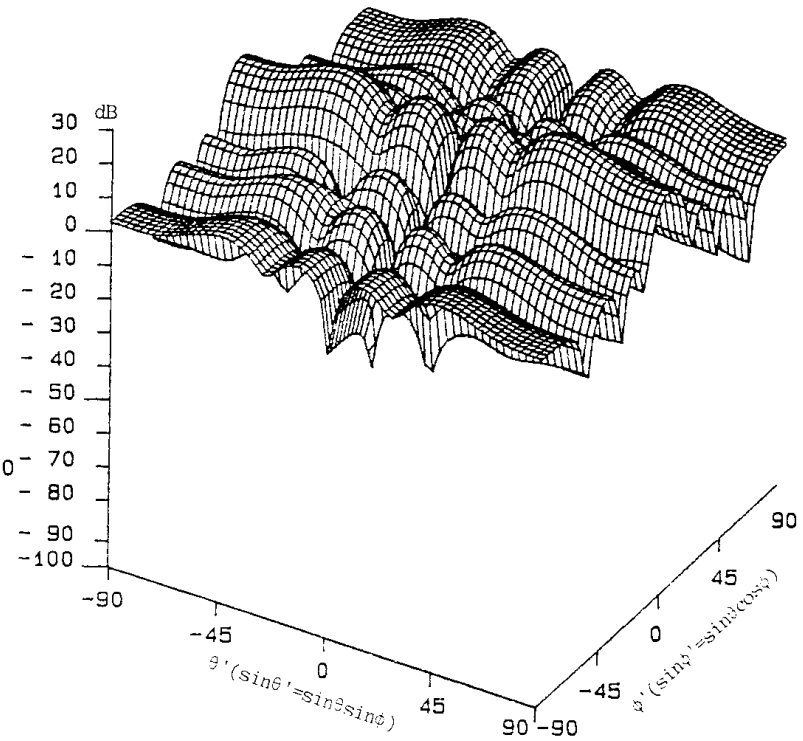

Figure 4 (a) The Array Beam Pattern in The Case of Position Error

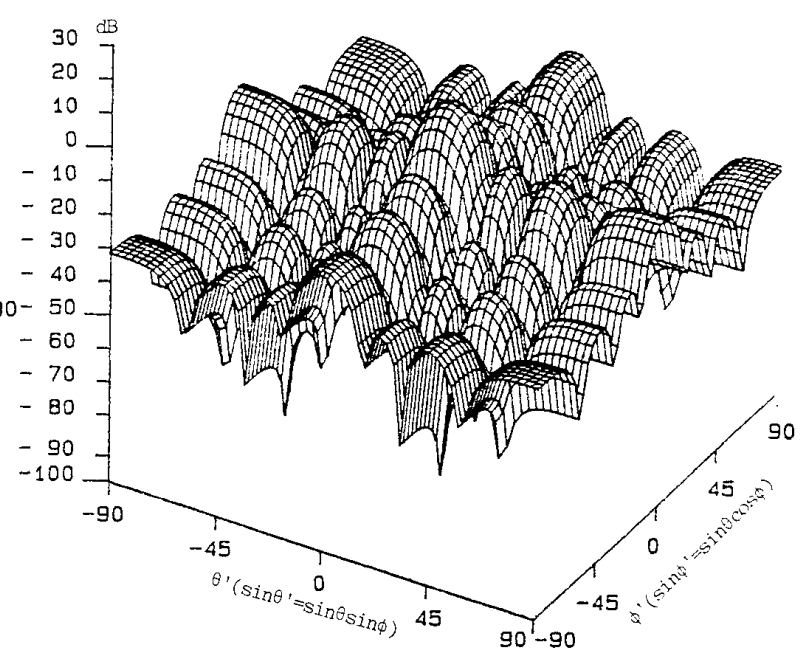

Figure 4 (b) The Array Beam Pattern After Robust Processing 